\title{
BUCHBESPRECHUNGEN
}

Bach, Maurizio: Europa obne Gesellschaft. Politische Soziologie der europäischen Integration.

(Peter Schimany)........................ 112

Gründinger, Wolfgang: Aufstand der Jungen. Wie wir den Krieg der Generationen vermeiden können.

(Bernd M. Malunat)....

Heyer, Andreas: Sozialutopien der Neuzeit. Bibliographisches Handbuch.

(Thomas Schölderle).

Mildt, Dick de (Hg.): Tatkomplex: NS-Euthanasie. Die ost- und westdeutschen Strafurteile seit 1945.

(Raimond Reiter).

Scheuch, Ute: Es musste nicht Soziologie sein, aber es war besser so. Erwin K. Scheuch - Eine Biographie.

(Klaus Hornung)...

Maurizio BACH: Europa obne Gesellschaft. Politische Soziologie der europäischen Integration, VS Verlag für Sozialwissenschaften, 2008, 207 Seiten, 19,90 EUR.

Europa ist in der öffentlichen Diskussion präsent wie nie zuvor. In der sozialwissenschaftlichen Europaforschung herrscht allerdings die Ansicht vor, dass in der Europäischen Union (EU) zwar die wirtschaftliche und politische Integration weit fortgeschritten ist, nicht aber der Prozess der sozialen Integration. Als Folge davon ist das Wissen über die Europäische Union marginal und eine »europäische Identität « nur schwach ausgeprägt. Dies wiederum schlägt sich in einer geringen $\mathrm{Zu}$ friedenheit mit den EU-Institutionen und in einer niedrigen Beteiligung an Europawahlen nieder. Vor diesem Hintergrund geht der Autor der Frage nach, wie sich im Zuge der »Europäisierung « die »europäische Gesellschaft « beschreiben lässt.

Die Entwürfe hierzu sind, wie einleitend betont wird, heterogen, diffus und umstritten. Sie basieren zumeist auf der Annahme, die Entstehung einer »europäischen Gesellschaft « sei grundsätzlich möglich. Hierbei orientieren sie sich an dem Gesellschaftsmodell des Nationalstaates. Gegenüber der Vorstellung, die europäische Integration fördere die Entstehung einer »europäischen Gesellschaft « nach dem Muster der Nationalgesellschaft, wendet der Autor jedoch ein, dass sich gerade im Verlauf des europäischen Integrationsprozesses die Grundlagen gesellschaftlicher Ordnungsbildung verändert haben. Der Europäisierungsprozess greife in die nationalstaatlichen Gesellschaftstypen ein und transformiere diese. In zahlreichen Politikfeldern - wie z.B. auch in der Asyl- und Migrationspolitik - beanspruchen Normen Geltung, die auf EU-Ebene ausgehandelt oder durch Rechtsprechung des Europäischen Gerichtshofes eingeführt wurden.

In makrosoziologischer Perspektive werden die gesellschaftlichen Folgen der politischen und ökonomischen Integration Europas aufgezeigt. Die acht Kapitel, umrahmt von Einführung und Ausblick, basieren weitgehend auf bereits veröffentlichten Arbeiten. Die unterschiedlich langen, aber immer gleichermaßen gehaltvollen Kapitel stehen in einem argumentativen Zusammenhang, können aber auch einzeln gelesen werden.

Den Ausgangspunkt bilden Ausführungen zur »Marktintegration ohne Sozialintegration " (Kapitel II). Danach wird die These formuliert, dass die mit der europäischen Integration eingeleitete Entgrenzungsdynamik nur aus der Institutionenperspektive angemessen begriffen werden kann (Kapitel III). Die institutionelle Dynamik der europäischen Integration wird dann mit Bezug auf die Folgen für die Demokratie (Kapitel IV) und auf die weitgehende Verselbständigung bürokratischer Herrschaft behandelt (Kapitel V). Die nachfolgenden Kapitel sind weniger stark durch eine theoretische und institutionelle Perspektive geprägt, vermehrt finden auch sozialstrukturelle und anwendungsorientierte Aspekte Eingang in die Analyse. 
Es folgt eine Analyse der räumlichen Dimension des europäischen Projekts, wobei insbesondere auf die vorletzte Erweiterungsrunde, die Aufnahme mittel- und osteuropäischer Länder im Jahr 2004, und deren Folgedynamik eingegangen wird (Kapitel VI). Die Erweiterung bzw. Entgrenzung Europas hat nicht nur eine Neustrukturierung des geographischen Raumes zur Folge, sondern im Kontext des Wanderungsgeschehens und neuer Migrationsformen auch eine Neudefinition von Staatsbürgerschaft und Einbürgerung (Kapitel VII). Mit der Formierung Europas als erweiterten transnationalen Raum treten zudem neue Konfliktlinien und soziale Ungleichheiten hervor; für eine Europasoziologie wirft dies theoretisch-konzeptuelle Probleme auf (Kapitel VIII).

Schließlich wird der Frage nachgegangen, ob die Herausbildung einer europäischen Identität die Legitimationsdefizite der EU kompensieren kann. Diese, so der Autor, kann unter Modernitätsbedingungen kein Ausweg aus den strukturell bedingten Widersprüchen der europäischen Integration sein (Kapitel IX). Im Ausblick wird dann noch einmal Bezug genommen auf das Risikopotenzial und die Krisenanfälligkeit der supranationalen Institutionenpolitik.

Die EU präsentiert sich als eine überlegene politische Ordnung, die Fortschritt und Solidarität verspricht und zu der es keine Alternative gibt. Kritik am Integrationsprozess widerspricht aus der Sicht der »Europabefürworter « der Vernunft der Geschichte. Die Kernaussage des Autors lautet jedoch, dass die EU weder über einschlägige Kompetenzen noch über notwendige Ressourcen sowie über hinreichende demokratische Legitimation verfügt, um die Sozialintegrationsverluste der Mitgliedsstaaten angemessen kompensieren zu können. Die EU befindet sich in einem Dilemma: Sie betreibt die Entwertung des Nationalstaats als »Normalform《 von Gesellschaft, ohne jedoch die europäische Ordnungsvorstellung mit gesellschaftlichem Leben erfüllen zu können.

Von der Entstehung einer den Nationalstaaten ähnlichen europäischen Gesellschaft kann somit keine Rede sein. Zwar gebe es eine Angleichung bestimmter Leitprinzipien, gemeinsame grundlegende Wertebezüge und politische Ordnungsideen würden aber fehlen. Eine fortgesetzte Erweiterung der EU verhindere zudem eine tiefere soziale Integration und die Identifikation der Bürger mit der EU. Identifikationsdefizite und Bürgerferne bergen aber die Gefahr der Entstehung eines neuen fremdenfeindlichen Nationalismus auf Seiten der Europäisierungsverlierer und Integrationsskeptiker.

Vor diesem Hintergrund muss man die EU als Sozialgebilde begreifen, das oberhalb der Nationalstaaten eine neue Form der Sozialintegration ausbildet. Diese durchdringt nur bestimmte Bereiche des sozialen Lebens, während andere Politikfelder auf nationaler Ebene verbleiben. Europa bzw. die EU ist nicht einfach nur eine Addition der verschiedenen Nationalstaaten und ihrer Geschichte. Gerade deshalb kann man die sozialwissenschaftliche Europaforschung nicht nur als Institutionensoziologie begreifen, wie das der Autor vorschlägt. Damit werden analytische Zugänge verengt. Untersuchungen sozioökonomischer und soziodemographischer Strukturen zeigen ja gerade die »Vielfalt in der Einheit «bzw. die sich überlagernden Formen von Sozialintegration auf nationaler und EU-Ebene.

Gleichwohl liest sich die Lektüre mit Gewinn. Der Autor vermittelt eine Reihe von Einsichten, die dazu anhalten, die gängige Europarhetorik und die EU als supranationalen Herrschaftsverband ebenso kritisch zu reflektieren wie vereinfachende empirische Befunde zur europäischen Integration. Vor allem aber verdankt sich der Lektüre die Erkenntnis, dass die Begriffe "Integration" und »Identität« im Kontext von Europa kritisch zu hinterfragen sind.

Peter Schimany

Wolfgang GRÜNDINGER: Aufstand der Jungen. Wie wir den Krieg der Generationen vermeiden können; Beck-Verlag, München 2009, 267 S.; 12,95 EUR.

Es ist durchaus zu erwarten, dass sich die Auseinandersetzung zwischen armen Jungen und reichen Alten, die Frage nach einem angemessenen Ausgleich zwischen den Generationen also, zu einem der zentralen Thema der deutschen Innenpolitik auswachsen wird. Schon jetzt ist die ältere Generation - nicht nur die der Rentner - privilegiert, der wachsenden Altersarmut zum Trotz. Die heutigen Rentner haben ein wenn schon nicht bekömmliches, so ein doch auskömmliches Einkommen, das die Kosten der Lebenshaltung abdeckt - zumeist jedenfalls. Die heute (noch) Beschäftigten haben 
Arbeit, mehr oder minder gute Verdienste und die Aussicht, sich später in eine Rente »retten « zu können - Privilegien, die die nachkommenden Generationen werden bezahlen müssen.

Das Dilemma zeichnet sich also deutlich ab. Die Jungen werde nicht nur für die Renten der Alten aufkommen, sondern zugleich auch noch ihre eigene Altersversorgung ansparen müssen - denn zukünftig wird die staatliche, richtiger die gesellschaftlich vereinbarte Rente nur noch einen kleinen Beitrag zur Altersversorgung leisten. Damit aber längst nicht genug. Viel schwerer wiegt, dass schon die gegenwärtige Generation der Schulabgänger nur noch verminderte Chancen hat einen Ausbildungs- bzw. Arbeitsplatz zu finden, also durch Arbeit zu Brot zu kommen - sie können sich daher selbst nicht oder nur unzureichend versorgen! Wie könnten sie da vorsorgen für sich und die Renten der Alten? Dabei sind die ökologischen Lasten der bereits abgetretenen Generationen, die weit heftiger ausfallen werden, als bisher verstanden, noch nicht einmal berücksichtigt.

Das Potential für einen Konflikt zwischen den Generationen ist also enorm, und es ist keineswegs ausgeschlossen, dass es zu einer offensiven Entladung kommen wird. Als Ursache kann man die Altersstruktur der Bevölkerung ausmachen, eine Bevölkerungs-Pyramide, die gewissermaßen auf dem Kopf steht, und dadurch das Problem auslöst, dass immer weniger Arbeitende für immer mehr Ruheständler aufkommen müssen. Man könnte allerdings auch auf die Idee verfallen, dass die Art und Weise, wie wir wirtschaften, den gesellschaftlichen Bedürfnissen - schon längst - nicht mehr entspricht. Es ist aber auch möglich, einfach sachlich zu analysieren, um zu realistischen politischen Vorschlägen zu gelangen, die das scheinbar riesige Problem dann ziemlich klein erscheinen lassen. Der medial beschworene »Krieg der Generationen « kann dann entfallen, es bleibt beim friedlichen und freundlichen Miteinander. Diesen sympathischen Ausweg oder Umweg beschreibt Wolfgang Gründinger auf überzeugende Art.

Der noch sehr junge Autor, gerade 26 Jahre alt, betrachtet zunächst die demographische Entwicklung. Er gelangt zu dem wenig überraschenden Ergebnis, dass eine aktive Bevölkerungspolitik notwendig ist, die allerdings nicht nur einer numerischen Erhöhung der Reproduktionsrate bedarf, vielmehr dort erst beginnen muss. Eben so wichtig ist nämlich, dem anzustrebenden Nachwuchs die bestmöglichen Chancen für dessen Zukunft zu eröffnen. Nicht nur in dessen Interesse übrigens, vielmehr in dem der gesamten Gesellschaft, weil nur bestens ausgebildete Menschen in der Lage sind, die wirtschaftliche Produktivität zu erhöhen. Dies aber ist eine entscheidende Bedingung, um im internationalen Wettbewerb bestehen zu können und um die geringeren Leistungen einer reduzierten Bevölkerung zumindest auszugleichen. Erforderlich ist allerdings auch, Zuwanderung zu fördern und für eine echte Integration zu sorgen, in einer Größenordnung von 100.000 bis 200.000 Menschen jährlich. Dennoch reduzieren sich viele der sozialen Kosten, weil eine kleinere Gesellschaft eben auch weniger Bedarf hat (an Straßen, Schulen, Infrastrukturen usw.). Die demographische Entwicklung - so ein Zwischen-Fazit - diente bisher vor allem als Vorwand, um die sozialen Leistungen zusammenzustreichen.

Stellt der demographische Wandel also kein unüberwindbares Problem dar, so werden auch die Sozialversicherungen nicht wirklich in Schwierigkeit geraten - jedenfalls lassen sich die gegenwärtigen Probleme nicht mit der sog. Überalterung begründen. Der Autor zeigt sehr eindringlich, dass und warum das bestehende Umlage-Verfahren der Sozialversicherungen - Rente, Gesundheit, Pflege, Arbeitslosigkeit - zuverlässig funktionieren kann, weit zuverlässiger jedenfalls, als es die hochgejubelte Alternative der kapitalgedeckten Privatversicherung jemals könnte. Um weiterhin erfolgreich zu bleiben, sind aber vielfältige politische Umsteuerungen erforderlich, die das System zugleich gerechter gestalten können und müssen. Deshalb sollte der Generationenvertrag korrigiert und so »gerettet« werden.

Um das Thema Generationen-Gerechtigkeit abzurunden, unternimmt Gründinger einen langen, gut verständlichen Exkurs in das volkswirtschaftliche Sachgebiet der Staatsverschuldung. Verschuldung ist so lange kein Problem wie in die Zukunft investiert wird, und damit auch in die Chancen der jungen Generation. Wo dringend gespart werden muss und kann, weist der Autor an einer Vielzahl von Positionen nach. Vor allem aber geht es ihm darum, durch höhere Einnahmen auch etwas mehr Steuergerechtigkeit herzustellen. Er gelangt zu dem Ergebnis, dass sich leicht 75 Milliarden Euro Mehreinnahmen erzielen lassen; dabei sind Verluste, die aus der Korruption entstehen, noch nicht einmal berücksichtigt. 
Der Autor schließt seine eingängigen Überlegungen mit einem Blick auf die »prekäre Generation «, auch »Generation Praktikum « genannt. An ihr zeigt sich die Doppelzüngigkeit der deutschen Politik und der deutschen Wirtschaft wie im Brennspiegel. Nachdem die Kinderlosigkeit der Deutschen ausgiebig bejammert wurde, werden die paar Kindlein, die das Licht der Welt dennoch hierzulande erblicken, umgehend als Ausschuss entsorgt: $\mathrm{Zu}$ wenige Kitas, schlecht ausgestattete Schulen und Universitäten, steigende Kosten, mangelnde berufliche Ausbildung, dafür aber fehlende Arbeitsplätze und zukünftig Berge von Schulden, finanzielle und ökologische. Noch schlechter lässt sich Deutschlands Zukunft politisch fast schon nicht mehr gestalten. Das schreit geradezu nach grundlegenden Veränderungen, und der »Aufstand der Jungen « könnte dazu ein wichtiger Schritt sein.

Demgegenüber zeichnet Wolfgang Gründinger ein sehr positives, dennoch ziemlich realistisches Bild. Er macht Mut - Jungen und Alten zugleich. Bedingung dafür allerdings ist, die Politik nicht nur in Worten, sondern Taten zukunftsfähig zu machen. Darin scheint mir der einzige Mangel der wirklich überzeugenden Schrift zu liegen: Der "Aufstand der Jungen « kommt nur im Titel vor, der Inhalt vermeidet dagegen jeden Hinweis auf einen bevorstehenden »Krieg der Generationen «, weil der Autor sehr optimistisch eine positive politische Entwicklung fordert. Dafür fehlen aber so gut wie alle Hinweise. Es geht noch immer nicht darum, vernünftig, über den Tag hinaus, nicht zuletzt auch zugunsten der Zukunft der Jungen zu entscheiden. Es geht nach wie vor immer noch nur um den Profit von heute - selbst die Banken-Krise, der dem Buch in die Quere kam, hat daran nicht das Geringste geändert. Vielleicht kommt es doch noch zu einem Aufstand!

Bernd M. Malunat

Andreas HEYER: Sozialutopien der Neuzeit. Bibliographisches Handbuch. Bd.2: Bibliographie der Quellen des utopischen Diskurses von der Antike bis zur Gegenwart (Politica et Ars; Bd.20). Münster 2009, LIT Verlag, 713 S., brosch., 69,90 EUR.

Die Utopieforschung hat in den letzten Jahren einen beachtlichen Aufschwung erlebt. Seit Anfang der 1990er Jahre sind eine Vielzahl an Monografien und Sammelbänden erschienen. Lieferte zunächst die Debatte um das »Ende der Utopie «- nach dem Ende des Realsozialismus - noch die entscheidenden Impulse, so hat sich der Diskurs davon inzwischen emanzipiert. Der eher oberflächliche Streit ist einem verstärkten Interesse an den Quellen und ideengeschichtlichen Traditionssträngen des utopischen Denkens gewichen. Verantwortlich für die konstruktive Wende sind nicht zuletzt zahlreiche Publikationen aus der Feder von Richard Saage und Andreas Heyer, die maßgeblich die analytische Überlegenheit eines als »klassisch « apostrophierten Utopiebegriffs nachgewiesen haben. Im Zentrum ihres Forschungsinteresses stand dabei von Anfang an, die Utopie als eigenständigen und klar umgrenzten Gegenstand zu etablieren.

Diesem Interesse folgt auch der zweite Band des »Bibliographischen Handbuchs « zu den Sozialutopien der Neuzeit von Andreas Heyer. Während sich der erste Band auf die Forschungsliteratur konzentriert, stellt der zweite Teil nun die "Quellen des utopischen Diskurses « in den Mittelpunkt. Ein dritter Band, der weitere Materialien und ein Gesamtregister enthalten wird, soll in Kürze folgen.

Um es vorweg zu nehmen: Heyer hat ein hochinteressantes, detail- und kenntnisreiches, manchmal auch etwas polemisch oder einseitig argumentierendes Kompendium vorgelegt. Dabei ist Heyers Sammlung erkennbar von der Intention getragen, sich den begrifflichen Entgrenzungstendenzen zu widersetzen und die Utopie von unterscheidbaren Formen wie Science-Fiction, Mythos, Eschatologie oder Robinsonade zu differenzieren. Der Umfang von gut 700 Seiten erklärt sich daher nicht aus einem ausufernden Begriffsfeld, sondern aus dem Konzept des Handbuchs selbst. Angesichts der enorm gewachsenen Recherchemöglichkeiten durch die modernen Informationstechnologien kann sich eine Bibliographie nicht mehr mit dem bloßen Sammeln von Katalogisaten zufrieden geben. Gefragt sich Kommentare, ideengeschichtliche Verortungen und Forschungsberichte, die die Literaturlisten ergänzen und strukturieren. Weil das in hohem Maße mühsam und zeitraubend ist, sind derartige Großprojekte selten geworden, zumindest, wenn sie den Anspruch erheben, für die Forschung einen wirklich nachhaltigen Nutzen zu stiften. Heyers Werk repräsentiert insofern tatsächlich eine Art Bibliographie neuen Typs. Den 
gesteigerten Anforderungen hat er sich mit aller Kraft gestellt. So ist der nun vorliegende Band 2 auch deutlich mehr ein Handbuch denn ein schlichtes Literaturverzeichnis.

Unterteilt ist der Band in drei Teile. Untersucht werden antike Quellen und ihr Verhältnis zum modernen Profil der neuzeitlichen Utopie, die in der Utopia von Thomas Morus unbestreitbar ihren Prototyp gefunden hat; ferner die Utopia selbst sowie ihre erstaunlich divergenten Interpretationsperspektiven; und schließlich der gesamte neuzeitliche Utopiediskurs in einer Auswahl von 100 Autoren und Schlagwörtern (z.B. »Frauenutopien «, »Idealstadtdiskurs «). Jedem der drei Teile ist eine umfangreiche Einleitung vorangestellt, ergänzt durch Kommentare oder Kurzporträts der Autoren, einer Auswahl exemplarischer Zitate, der Angabe von Editionen und der entsprechenden Forschungsliteratur.

Abermals verwunderlich - wie schon in Band 1 - ist allerdings die Tatsache, dass dieses Handbuch zu den "Sozialutopien der Neuzeit « die Quellen (laut Untertitel) »von der Antike bis zur Gegenwart « aufzuschlüsseln versucht. Dieser Widerspruch wird - anders als noch in Band 1 - diesmal eingehend thematisiert. Neben dem Verfasser widmet sich auch Richard Saage in seinem Geleitwort (5-7) diesem Punkt. Das Vorgehen erklärt sich dabei aus der Überzeugung, dass die neuzeitliche Utopie zwar ein durchaus eigenständiges Profil besitzt, den antiken Texten gleichwohl eine wichtige Vorläuferfunktion zukommt. So zutreffend diese Einschätzung ist, so sehr macht sie im Grunde den Verweis auf »Neuzeit« überflüssig. Man hält tatsächlich ein Handbuch zum Utopiediskurs von der Antike bis zur Gegenwart in Händen. Andreas Heyer widmet sich immerhin knapp 130 Seiten lang der Frage nach dem antiken Vorbildcharakter. Positiv formuliert: Das Buch hält mehr als der Hauptsachtitel verspricht.

Der Haupteinwand gegen das Konzept bzw. die Systematik des Bandes liegt aber - auch auf den zweiten Blick - in der irritierenden Zuordnung bzw. Aufteilung zwischen den Bänden: Wenn Utopien ausdrücklich durch ihre »Doppelstruktur von Gegenwartskritik und utopischer Alternative« (269) definiert werden, dann zeichnen sich die Quellen eben durch die Existenz eines Entwurfs alternativer Gesellschaftswirklichkeit aus und unterscheiden sich dadurch genuin von der »Utopietheorie«, die diese Entwürfe rekonstruiert, analy- siert sowie methodisch und begrifflich zu erfassen versucht. Insofern muss rätselhaft bleiben, weshalb letztlich auch »einschlägige Texte der Utopietheorie « (269) nahtlos in die Quellensammlung mit aufgenommen sind. So finden sich im eigentlichen Hauptteil (\$10) Autoren wie Mohl, Landauer, Mannheim, Bloch oder Popper in einer Reihe mit Klassikern wie Campanella, Bacon oder Mercier. Diese aber können im eigentlichen Sinn nicht den Primärquellen zugerechnet werden. Sie stehen vielmehr sogar maßgeblich für die drei unterschiedlichen Ansätze in der Begriffsdiskussion (weshalb man sie also in Band 1 vermuten würde): Mohl ist Urvater des "klassischen Utopiebegriffs ", Landauer, Mannheim und Bloch sind die Protagonisten eines "sozialpsychologischen Ansatzes" und Popper ist der Begründer » totalitarismustheoretischer « Utopiekritik. Die gewählte Systematik muss beim Nutzer zwangsläufig den Eindruck erwecken, die genannten Autoren (aber auch Adorno, Picht oder Flechtheim) hätten ihrerseits eigenständige utopische Entwürfe zu Papier gebracht. Genau das aber ist nicht der Fall.

Zwar mag es aus strategischer Sicht durchaus Sinn ergeben, den Utopisten neben Werkeditionen auch die entsprechende Forschungsliteratur beizuordnen, doch zugleich die Utopieforscher in diese Reihe einzugliedern, sprengt den Ansatz (zumal ein eigener Band zur »Forschungsliteratur « existiert). Auch bei anderen Denkern wundert man sich gelegentlich über ihre Aufnahme in die Reihe der utopischen Quellen, so z.B. bei Montesquieu, über den der Verfasser selber urteilt, es gehe diesem primär um »empirisch-vergleichende Analysen des Bisherigen « (589), aber auch bei Machiavelli (556-559), der dem utopischen Denken gemeinhin als ziemlich unverdächtig gilt. Fairerweise muss man hinzufügen, dass der Handbuchcharakter für einige Klarstellung sorgt und unter anderem plausibel macht, dass die Aufnahme solcher Denker nicht zuletzt der Grenzziehung zum utopischen Denken dienen soll. Kurios bleibt trotzdem, warum beispielsweise Machiavelli beständig mit dem »neuzeitlichen Kontraktualismus « in Verbindung gebracht wird (270).

Zudem hätte man sich gelegentlich etwas mehr Sorgfalt gewünscht. So heißt beispielsweise der Herausgeber des Bodin-Sammelbandes nicht »Helmut«, sondern »Horst « Denzer (220); der Vorname von Mumford lautet "Lewis«, nicht »Louis« (245); und die Morus-Monographie von 
Reinhold Baumstark erschien »1879«, nicht »1979«(212). Ferner ist die Zitierweise zuweilen wenig leserfreundlich (die Angabe »a.a.O.« zieht bei fortgeschrittener Fußnotenzählung zwangsläufig langes Blättern nach den vollständigen Belegstellen nach sich). Bei Artikeln und Aufätzen fehlen häufig entsprechende Seitenangaben und mit Vornamen wird generell uneinheitlich verfahren, mal sind sie abgekürzt, mal ausgeschrieben angegeben. Darüber hinaus sind zum Teil recht relevante Lücken zu beklagen: Im Morus-Kapitel fehlt z.B. die einschlägige Biographie von Peter Berglar (»Die Stunde des Thomas Morus. Einer gegen die Macht «) oder der 6. Band (»Trostgespräche im Leid «) der deutschen Thomas-MorusWerke. Aber auch der Setzer hatte keinen allzu guten Tag: Es wimmelt nur so von »Hurenkindern « und "Schusterjungen « (besonders häufig: Überschrift auf der einen Seite, zugehöriger Text auf der nächsten). Orthographie, Interpunktion und Silbentrennung wären ebenfalls ein lohnendes Feld für eine erneute Redaktion.

Gleichwohl: Trotz der formalen und systematischen Mängel muss das Fazit positiv ausfallen. Heyer hat sich einer Aufgabe gestellt, an die sich für gewöhnlich wohl nur Autorenteams wagen, er hat völlig neue Pfade der Literaturdokumentation beschritten und dabei insgesamt ein Werk vorgelegt, dass in seiner Dichte und seinem Materialreichtum außergewöhnlich ist. Nicht alltäglich ist allerdings auch - das als Pointe zum Schluss -, dass sich der Verlag auf seinem eigenen Papier vom $\mathrm{Au}$ tor als Ursache für dessen schlechte Laune beschimpfen lässt (20).

\section{Thomas Schölderle}

Dick de MILDT (Hg.): Tatkomplex: NS-Euthanasie. Die ost- und westdentschen Strafurteile seit 1945. 2 Bände. 844 und 903 Seiten. Gebunden. Personalisierte PDF-Version beider Bände. Amsterdam 2009, 195,00 EUR.

Tatkomplex NS-Euthanasie ist eine Sammlung von Gerichtsurteilen. Sie betreffen deutsche Beschuldigte, die sich 1946 bis 1988 vor deutschen Gerichten wegen Tötungsdelikten in der Anstaltspsychiatrie zu verantworten hatten. Viele der Beschuldigten wurden als Täter verurteilt, einige in den ersten Nachkriegsjahren sogar zum Tode. $\mathrm{Zu}$ den 13 Todesurteilen aus den Jahren 1946 bis 1947 gehörte das gegen Professor Hermann Nitsche. Es hat auch eine Reihe von Freisprüchen gegeben.

Die in zwei Bänden auf 1.747 Seiten dokumentierten 42 Urteile lassen das System der NS-Verbrechen in der Psychiatrie umfassend und in vielen Details erkennen. Eine wertvolle Quellensammlung, auch wenn man der Sicht der Richter nicht immer folgen mag. Die Anklage hat aber in der Regel die historischen Umstände, die Tatbedingungen des NS-Staates, die zentral Verantwortlichen sowie die Tatbeiträge und Motivation festgehalten. Es sind hierdurch Tätergruppen erkennbar und ihre juristische Würdigung. Wer allerdings vermutet, dass diese Gerichtsurteilssammlung nur für Juristen interessant ist, wird überrascht sein. Denn die Inhalte sind in vielfältiger Weise relevant. Sie bieten u. a. regionalgeschichtliche Dimensionen und Dokumente zur Diskussion über den Charakter der NS-Herrschaft und ihre Terrororganisationen.

Die besondere Bedeutung des Tatkomplexes NS-Euthanasie kann nicht zuletzt darin gesehen werden, dass er das System der Geheimhaltung der NS-Herrschaft direkt betrifft, das erst in jüngerer Forschung weitergehender untersucht wird. (vgl. Raimond Reiter, Hitlers Geheimpolitik, Frankfurt am Main 2008).

Die »T4-Aktion« mit ca. 71.000 Opfern und die »Kinder-Aktion« mit über 5.000 Opfern waren »Geheime Reichssachen «. Ebenfalls streng geheim bleiben sollten andere Tötungsaktionen an Anstaltsinsassen wie die so genannte "wilde Euthanasie « und die "Aktion $14 \mathrm{f} 13$ «. Zu diesen und weiteren Fällen bieten die Gerichtsurteile umfangreiches Material einschließlich historischer Dokumente. Überhaupt kann in der Dokumentation von Gerichtsurteilen zu NS-Verbrechen eine der zentralen Quellenarten gesehen werden, da uns sonst durch Geheimhaltungspolitiken der Nationalsozialisten ebenso wie systematische Aktenvernichtungen noch kurz vor Kriegsende vieles nicht überliefert wäre (zur Geheimhaltung: Band 1, 390-395). Die Gerichtsurteile und die dazugehörigen Ermittlungsakten in den jeweiligen Archiven sind also für viele Forschungsdisziplinen von groBer Bedeutung.

Dem kundigen Leser ist wohl bekannt, dass die westdeutschen Urteile zu NS-Verbrechen in der Psychiatrie bereits in der Urteilssammlung "Justiz und NS-Verbrechen " veröffentlicht worden sind. Aus dieser Sammlung stammt auch die Einteilung 
der NS-Verbrechen in 12 Deliktgruppen und die Bezeichnung »Euthanasie«, unter die etwa $5 \%$ aller westdeutschen Urteile fallen. Die jetzt gebündelte Veröffentlichung der gesamten Urteilsgruppe bietet einen einfachen vergleichenden Zugang. Der Herausgeber Dr. Dick de Mildt hält weitere nach Tatkomplexen gebündelte Veröffentlichungen für vorstellbar, also für Schreibtischverbrechen, Justizverbrechen usw.

Welche inhaltlichen Dimensionen lassen sich in der neuen wichtigen Veröffentlichung finden bzw. herausheben? Eigentlich ist das gesamte Specktrum der Themen zu erkennen, die auch in der Forschung dargestellt sind. Zunächst gehört dazu das von Hitler und seiner Reichskanzlei initiierte »T4«-Programm und dessen Organisationen (exemplarisch: Band 1, 40-44; 180-191. Band 2, 549ff.)

Wichtig ist die Rolle der » $\mathrm{T} 4$ «-Zentrale in Berlin, die auch nach dem Ende der »T4-Aktion « 1941 bei weiteren Tötungsaktionen wirksam war (Band 1,353ff. Band 2, 209ff). Zur reichsweiten Organisation des Massentötens gehörten Dokumente, die in Urteilen zu finden sind: Hitlers Erlass vom 1. September 1939, die »Meldebogen « zur Aussonderung zu tötender Patienten, Standardschreiben zur Tarnung des bürokratischen Tötens usw. (Band 1, 393ff. Band 2, 852ff).

Es hat im Zweiten Weltkrieg sechs zentrale Vernichtungsanstalten gegeben. Bis auf Hartheim, da bei Linz in Österreich gelegen, treten alle auf, einige in mehreren Urteilen: Grafeneck (BadenWürttemberg), Brandenburg an der Havel (Brandenburg), Sonnenstein (Pirna, Sachsen), Bernburg Saale (Sachsen-Anhalt) und Hadamar (Hessen).

Ein weiterer großer Komplex betrifft die »Kinderfachabteilungen «, in denen geistig und körperlich behinderte Kinder getötet wurden. Auch hier haben Gerichte das System der Verbrechen in Betracht gezogen, um die Tatbeiträge der Einzeltäter juristisch würdigen zu können (Band 1,110f). Die juristischen Einordnungen sind allerdings nicht immer leicht verständlich (Befehl und Gehorsam, Befehlsnotstand, Schuldausschließungsgrund usw.). Es hat über 30 »Kinderfachabteilungen« und ähnliche Einrichtungen gegeben, ihre genaue Zahl ist bis heute nicht gesichert. Die Gerichtsurteilssammlung kennt vor allem Details zu den Beispielen Kalmenhof, Eglfing-Haar, Sachsenberg, Waldniel und Wiesloch.

In einigen Urteilen wird näher auf die so genannte "wilde Euthanasie« eingegangen, der ver- mutlich weit über 200.000 Patienten zum Opfer gefallen sind (Band 1, 3ff, 397) und die "Durchgangs- und Sammelanstalten «, die der Tarnung und der geordneten Zuführung der Patienten zu Tötungsstätten dienten (Band 1, 39ff, 381ff, 445ff). $\mathrm{Zu}$ den besonderen und gelegentlich übersehenen Opfergruppen gehören die jüdischen Anstaltspatienten (Band 1, 403), die der "Sonderbehandlung $14 \mathrm{f} 13$ « (Band 2, 653ff) und die »Ostarbeiter « in der Anstaltspsychiatrie (Band 2, 675ff). Für diese Patientengruppen gab es besondere Sammelanstalten, um sie zu töten.

Besonders interessant sind Urteile, in denen es um die Frage ging, ob Beschuldigte sich kritisch oder gar oppositionell gegen das »Euthanasie«Programm verhalten haben. Mit diesem Komplex haben sich insbesondere die Landgerichte Düsseldorf und Hannover befassen müssen (Band 1, 687ff. Band 2, 95ff). Tatsächlich hat es hier eine Reihe von Freisprüchen gegeben. Wie in anderen Deliktgruppen auch war die Feststellung strafrechtlich zu ahnender Taten bei "Schreibtischtätern « nicht einfach und nicht unstrittig. So hatten sich die drei Angeklagten der früheren Provinzialverwaltung in Hannover nach Auffassung des Landgerichts Hannover zwar der Beihilfe zum Mord schuldig gemacht. Dennoch hatten sie durch ihr verharren im Amt vermeintlich versucht, "zu retten, was zu retten war«. Sie waren zudem innerlich gegen die von ihnen aus Berlin verlangten Patientenverlegungen zu Tötungsanstalten eingestellt und durch die Umstände des nationalsozialistischen Staates käme deshalb bei Würdigung aller Umstände ein »übergesetzlicher Schuldausschließungsgrund « zum tragen (Band 2, 150).

Die Materialfülle der Urteilstexte ist mit den genannten Dimensionen nicht erschöpft. So finden sich Passagen zu einigen bekannten Tätern, u.a. zu: Philipp Bouhler, Viktor Brack, Werner Heyde, Friedrich Mennecke, Hermann Nitsche, Hans Hefelmann und Hermann Pfannmüller. Auch gibt es in einigen Urteilen Namen von Opfern (Band 1, 395. Band 2, 116, 518ff, 677).

Die Nutzungsmöglichkeiten des neuen Werkes sind vielfältig. Für einige historische Forschungen wird es eher ergänzendes Material sein, da viele Themen schon bearbeitet sind. Gleichwohl verweisen die einzelnen Urteile auf teilweise große Aktenbestände in Archiven. Regionalgeschichtliche Bezüge können ebenso von Bedeutung sein wie Opfernamen, die für das Gedenken interessant 
sein können. Ebenfalls Neues kann sich aus einem Ost-West-Vergleich der Rechtsprechung zu NSVerbrechen ergeben. Dies betont auch der Herausgeber im Vorwort, in dem er die Urteile als einzigartige Quelle hervorhebt, »... berichten sie doch nicht nur über Euthanasie-Verbrechen sondern auch über den Umgang mit diesen Verbrechen in den Jahren nach 1945 in zwei von unterschiedlichen Gesellschaftssystemen geprägten deutschen Staaten.«(Band 1, V)

Das Register umfasst Tatorte, Angeklagte, beteiligte Dienststellen usw. Hinzu kommt eine PDF-Version, die als personalisiertes Dokument heruntergeladen und passwortgeschützt genutzt werden kann. Mit diesem zeitgenmäßen Service ist eine Volltextsuche problemlos möglich.

\section{Raimond Reiter}

Ute SCHEUCH: Es musste nicht Soziologie sein, aber es war besser so. Erwin K. Scheuch-Eine Biographie. Gerhard Hess Verlag, Schussenried 2008,448 Seiten, 36,00 EUR; dies: Erwin K.Scheuch im roten Jahrzehnt. Edwin Ferger Verlag, Bergisch Gladbach, 2008, 212 Seiten, Hardcover 28,00 EUR, brosch. 18,00 EUR.

In den 60er und 70er Jahren des vorigen Jahrhunderts gehörte Erwin K. Scheuch mit Ralf Dahrendorf, Helmut Schelsky, Rene König und anderen zu den bekanntesten deutschen Soziologen. Sein Leben und vor allem sein akademisches und wissenschaftliches Wirken hat nun die Ehefrau des 2003 Verstorbenen in zwei gründlich recherchierten Bänden nachgezeichnet. Scheuch wurde 1928 in einer bewusst katholischen Familie in einfachen Verhältnissen in Köln geboren, der Vater war zu dieser Zeit arbeitslos. Er begann das Studium der Soziologe 1949 in Köln bei dem Altmeister Leopold von Wiese, mit dessen Empfehlung er schon 1950 an einem der ersten akademischen Austauschprogramme in den USA teilnehmen konnte. An der Universität Connecticut lernte er die gerade in Blüte gekommenen neuen Methoden der empirischen Sozialwissenschaft kennen - des Interviews, der Umfragen und der Meinungsforschung, die Schwerpunkte seiner eigenen wissenschaftlichen Arbeit werden sollten. Schon 1956 promovierte er in Köln bei Rene König, dessen Assistent er wurde und der ihn in die Netzwerke der internationalen wissenschaftlichen Gemeinschaft ein- führte. So konnte er bereits 1956 am 3. Weltkongress der Internationalen Gesellschaft für Soziologie in Amsterdam teilnehmen. 1962 folgte die Einladung auf eine Gastdozentur in Harvard und damit der Durchbruch zur Internationalität. Seine Habilitationsarbeit über Messverfahren in den Sozialwissenschaften eröffnete ihm mit 37 Jahren eine ordentliche Professur in Köln, wo er zu einem der wichtigsten Repräsentanten der jungen empirischen und quantifizierenden Sozialwissenschaften in der Bundesrepublik wurde, die er in einer Vielzahl von Publikationen zu verbreiten versuchte, nicht ohne manchen Widerstand aus der älteren Generation, die, wie etwa Arnold Bergstraesser, den Vorrang der geisteswissenschaftlich-historischen Soziologie zu behaupten versuchten.

Bei der 16. Tagung der Deutschen Gesellschaft für Soziologie (DGS) im April 1968 in Frankfurt hatte Scheuch das Hauptreferat "Methodische Probleme gesamtgesellschaftlicher Analysen « übernommen. Es wurde zum Anstoß eines Methoden- und Prinzipienstreits, der unter dem Rubrum »Spätkapitalismus oder Industriegesellschaft? « in die Geschichte der deutschen Soziologie eingegangen ist. Scheuch richtete seine Kritik an die Adresse einer »Kulturintelligenz « und ihrer Sozialphilosophie, deren allgemeinen Deutungsschemata er als Ideologie kennzeichnete und der er die Soziologie als Erfahrungswissenschaft gegenüberstellte. Mit anderen Worten: Hier trafen die Frankfurter Schule von Adorno, Horkheimer, Habermas und die sogenannte neopositivistische Schule aufeinander, die keine endgültigen ontologischen Erkenntnisse anerkannte und insbesondere den Anspruch der Frankfurter entschieden ablehnte, die »wahren Bedürfnisse« der Gesellschaft zu kennen und zu bestimmen.

Der Konflikt bei der 16. Jahresversammlung der DGS stand im Zusammenhang mit der begonnenen Studentenbewegung in der Bundesrepublik. Der Frankfurter Sozialistische Studentenbund (SDS) hatte eine öffentliche Diskussion zwischen seinen Vertretern und Professoren der DGS erzwungen, bei der Scheuch und Dahrendorf die letzteren zu vertreten hatten. Zutreffend charakterisierte Günter Zehm in der Welt vor allem die Rolle Scheuchs bei dieser Diskussion als die eines »Winkelrieds « gegen die dogmatisch bornierten Studenten. Nach anfänglichen Vermittlungsversuchen machte Scheuch nun kompromisslos deutlich, dass er die Ziele und Methoden der Studen- 
tenbewegung für totalitär hielt. In seinem Bericht über die Diskussion tauchten jetzt Begriffe auf wie "Sportpalastatmosphäre « und "Tugendterror «, wissenschaftliche Veranstaltungen sollten $\mathrm{zu}$ »teach-ins in Vulgärmarxismus " umfunktioniert werden. Der Liberale Scheuch näherte sich Urteilen seines Kollegen Helmut Schelsky, der von dem hier aufgebrochenen Konflikt zwischen »wirklichkeitsverpflichteter und heilsgewisser Wissenschaft « sprach und die Frankfurter Schule als »neue Säkularreligion « mit quasireligiösen Zügen kennzeichnete.

Auch in dem 1968 erschienenen Sammelband unter Scheuchs Herausgeberschaft Die Wiedertäufer der Wohlstandgesellschaft - Eine kritische Untersuchung der Neuen Linken und ibrer Dogmen wurden nun die Grenzen gegenüber der Neuen Linken deutlich abgesteckt: Hier entwickelte sich eine Bewegung, die weit hinausging über die Fragen vernünftiger Reformen in Universität und Gesellschaft, eine Bewegung, die vielmehr an die Täufer in der Reformationszeit erinnerte, an ihren Chiliasmus und an ihr Ziel des "neuen Menschen «. Wenn Scheuch hier mit treffenden Formulierungen die Neue Linke als eine sich »auf Offenbarung berufende Erweckungsbewegung « mit dem »vorwissenschaftlichen Wunsch nach Totalerklärung « charakterisierte, kam dabei nicht nur sein Kriterium von Erfahrungswissenschaft ins Spiel, sondern konnten dem auch freiheitliche Konservative ohne weiteres zustimmen.

Durch die bald darauf erfolgende Gründung des »Bundes Freiheit der Wissenschaft « (10. November 1970), bei der Scheuch wiederum eine initiative Rolle spielte, wurde deutlich, dass sich hier eine Koalition sozialdemokratischer Reformer (Hermann Lübbe, Thomas Nipperdey, Richard Löwenthal u. a.) mit Konservativen (Hans Maier, Friedrich Tenbruck, Gerhard Löwenthal u. a.) zusammenfand in der Abwehr einer Politisierung der Wissenschaft durch den Neomarxismus und der Neuen Linken als einer Ideologie zur Verwirklichung utopischer Ziele und mit totalitären Methoden, die sich allesamt gegen die freiheitliche Demokratie des Grundgesetzes richteten. Diese Gründung wie auch Publikationen wie Die Wie- dertäufer der Wohlstandsgesellschaft haben damals das ihre dazu beigetragen, die Stoßkraft der linken Kulturrevolution zu brechen.

Zwischen 1971 und 1977 wurde Erwin Scheuch Zielscheibe fortgesetzter Störungen seiner Kölner Lehrveranstaltungen, war er doch als beredter Pressesprecher des Bundes Freiheit der Wissenschaft der sich radikalisierenden Studentenbewegung besonders verhasst. Diese planmäßigen Störungen der Lehrveranstaltungen missliebiger Professoren und ihre »Umfunktionierung « zu Agitprop- Veranstaltungen im Sinne des Stamokap, also des platten Parteimarxismus der DDR und der Sowjetunion, waren seinerzeit an den deutschen Hochschulen verbreitete Methoden zur Destabilisierung des »Spätkapitalismus « und blieben doch weithin ohne große Beachtung in der Öffentlichkeit und zumeist auch in der Politischen Klasse. Bei Scheuch und bei nicht wenigen anderen Professoren hinterließen sie nicht geringe gesundheitliche Schäden. Auch in Köln versuchten, wie damals häufig, »Feuilleton- und Parteimarxisten « in Professorenstellen und Lehrtätigkeiten der Universitäten einzudringen, Versuche, denen sich Scheuch wiederum entschieden widersetzte. In diesem Zusammenhang ging auch Scheuchs langjährige Freundschaft mit seinem akademischen Lehrer Rene König zu Bruch, auch dies einer der »Kollateralschäden « des »roten Jahrzehnts «, wie sie sich damals vielfach an deutschen Universitäten zutrugen.

Ute Scheuchs Biographie Erwin K. Scheuchs verdient Beachtung, zum einen als ein quellengesättigter Beitrag zur Wissenschaftsgeschichte der Bundesrepublik in der zweiten Hälfte des vorigen Jahrhunderts, zum anderen als Dokumentation der »Kulturrevolution « der sechziger und siebziger Jahre und ihre Spiegelung in der Lebensgeschichte eines herausragenden Hochschullehrers und in den universitären Institutionen. Manches von damals wirkt bis heute fort, und Ute Scheuchs Darstellung ist geeignet, auf die Wurzeln mancher Fehlentwicklungen der Gegenwart erneut aufmerksam zu machen.

Klaus Hornung 